\title{
Effect of Redundancy on Mean Time to Failure of Wireless Sensor Networks
}

\author{
Anh Phan Speer Ing-Ray Chen \\ Virginia Tech \\ Department of Computer Science \\ \{nphan, irchen\}@vt.edu
}

\begin{abstract}
In query-based wireless sensor networks (WSNS), the system must perform data sensing and retrieval and possibly aggregate data as a response at runtime. Since a WSN is often deployed unattended in areas where replacements of failed sensors are difficult, energy conservation is of primary concern. While the use of redundancy is desirable in terms of satisfying user queries to cope with sensor faults, it may adversely shorten the lifetime of the WSN, as more sensor nodes will have to be used to answer queries, causing the energy of the system to drain quickly. In this paper, we analyze the effect of redundancy on the mean time to failure (MTTF) of a WSN in terms of the number of queries the system is able to answer correctly before it fails due to either sensor faults or energy depletion. In particular, we analyze their effect of redundancy on the MTTF of cluster-structured WSN for energy conservations. We show that a tradeoff exists between redundancy and MTTF. Furthermore, an optimal redundancy level exists such that the MTTF of the system is maximized.
\end{abstract}

\section{Introduction}

Applications such as security and surveillance monitoring, battlefield command and control, and wildlife or medical monitoring rely on the correct functioning of the underlying WSN in data sensing and retrieval in response to application queries. We consider two major sources of faults that could cause the system to fail. One source is energy depletion of sensor nodes such that the WSN simply exhausts its energy to be able to answer queries. In such applications, the WSN is often deployed in an area where replacements of sensors are difficult or impossible. Another source is sensor faults including measurement faults.

To conserve energy of sensor nodes, a well accepted approach is for the WSN to self-organize itself into clusters. Within a cluster, a cluster head is elected to perform more data aggregation and relay duties than normal sensor nodes and is rotated among sensor nodes in the cluster for balancing energy consumption. An intracluster routing tree is maintained dynamically in response to sensor faults. For long-haul networks, cluster heads may relay data cluster-by-cluster to reach a processing center (e.g., where a user query is issued). Moreover, for applications concerned with sensor readings such as the minimum/maximum/average of sensor data, cluster heads can also perform in-network data aggregation and compression functions to reduce energy consumption [6]. A cluster can be reconfigured globally by the system for optimization purposes or in a distributed manner on a persensor-node basis [5]. To cope with the second source of faults, i.e., sensor faults, a general approach is to incorporate redundancy to allow sensor faults to be detected, isolated, and corrected so that the system can continue to function correctly in data sensing and retrieval. However, the use of redundancy impacts the energy consumption rate of the system since more sensor nodes would need to be used as redundancy to achieve sensor fault tolerance. Therefore, there is a tradeoff between these two sources of faults. On the one hand, we like to incorporate redundancy to deal with sensor faults. On the other hand, redundancy should be used only as needed so as not to quickly deplete the energy of the system.

Current research work on fault tolerance mechanisms to cope with sensor faults in WSNs can be classified into hardware redundancy, time redundancy and information redundancy $[1,2,6,7,8]$. This paper concerns with hardware redundancy which utilizes extra hardware for fault detection or masking. A sensor can be made to disambiguate a sensor measurement fault from a true event by using a distributed Bayesian algorithm $[6,7]$ after comparing readings obtained from its neighbor sensors of the same type. At the processing center (i.e., user query) end, more than one sensor reading responding to a query can be propagated back to the processing center via multiple paths, from which a voting can be performed using the first three readings. A majority reading, if it exists, will be passed to the application as the legitimate response. This approach was suggested in [2] for data fault tolerance of data propagation in WSNs.

In this paper, we investigate the effect of redundancy on the mean time to failure (MTTF), which is used as a metric to measure the effectiveness of the underlying WSN to answer user queries. We consider hardware redundancy in this paper, specifically, path redundancy $[2,3$, and 4$]$ and source redundancy to cope with sensor faults. We consider a WSN as having experienced a failure when it fails to deliver sensor data correctly in response to an application-level query, either due to energy depletion (i.e., the first source of faults) or due to sensor faults (i.e., the second source of faults). We show that using excessive redundancy to achieve fault tolerance toward sensor faults will have detrimental effects on the MTTF of the system. Given a WSN system characterized by a set of parameter values as identified in the paper, we 
show that there exists an optimal level of redundancy under which the MTTF is maximized. The contribution of the paper lies in the mathematical analysis and pioneering work on the tradeoff analysis between fault tolerance and energy consumption for WSNs.

\section{System Model}

A WSN consists of a set of low-power sensor nodes typically deployed through air-drop into a geographical area. The sensors in the network are indistinguishable with the same initial energy level. Sensors group themselves into distinct clusters in the system for energy conservation purposes, with each cluster ideally responsible for a feature area, as illustrated in Figure 1. Each cluster elects a sensor to be the cluster head. The role of a cluster head is rotated fairly among sensors in the cluster based on a cluster head rotation algorithm such as HEED [10] or LEACH [13] so that sensors will not quickly deplete their energy. The function of a cluster head is to manage the network within the cluster, and collect/aggregate sensor reading data from the sensors within the cluster. To save energy, the transmission power of a sensor even when it is a cluster head is reduced to a minimum level to enable it to communicate with its neighbor within one-hop radio range. Thus, a sensor needs to use a multi-hop route (i.e. passing through a number of other sensors) for it to communicate with another sensor node distant away.

We assume that users (through a flying airplane or a moving vehicle) can issue a query through any cluster head, which we call it a processing center or a user monitoring node as labeled in Figure 1. A query may involve all or a subset of clusters, say, $k$ clusters, to respond to the query for data sensing and retrieval. These involved clusters are termed source clusters. Our system does not have a base station. Also sensors in a cluster will rotate to be the cluster head in their cluster. Thus, the notion of higher energy consumption by critical nodes [14] for relaying messages to a base station or to a cluster head does not exist.

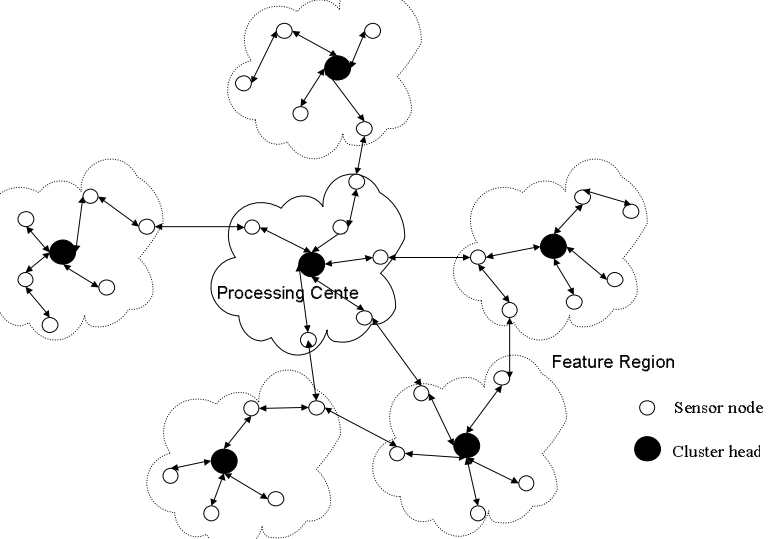

Figure 1: Cluster-based WSN architecture.
In a cluster-based architecture, a source cluster head must relay sensor data information to the processing center in response to a user query, and thus will consume more energy than a pure sensor node. The energy consumed by a source cluster depends on the length of the path connecting the source cluster and the processing center. For fault tolerance reasons, two forms of redundancy are considered for which we analyze their effect on MTTF in this paper. The first one is path redundancy. That is, instead of using a single path to connect a source cluster to the processing center, $m$ disjoint paths (where $m \leq 3$ practically) may be used. The second is source redundancy. That is, instead of having one sensor node return requested sensor data, $m_{s}$ sensor nodes within a source cluster may be used to return readings to cope with sensor hardware and software faults.

We characterize the failure behavior of a sensor due to environment conditions (i.e. hardware failure) by a failure probability parameter $q$. Assume that all sensors are deployed in a square sensor area of size $A^{2}$ such that each side is of length $A$. Also assume that the sensors in the network are distributed according to a homogeneous spatial Poisson process with intensity $\lambda$. Let $n$ be the total number of nodes in the WSN. Let $n_{s}$ be the number of sensors in a cluster. The size of a cluster depends on the clustering algorithm employed and is a design parameter that will affect the MTTF of the system. The number of clusters in the system, $N_{c}$, is given by $n / n_{s}$. A cluster head will perform the function of data collection, aggregation and communication. Let $E_{o}$ be the initial energy of each sensor node in Joule.

A user query may demand up to $k$ clusters to respond to it, where $k$ is in the range of 1 to $N_{c}$. When a query calls for a cluster to retrieve sensor data, one sensor is sufficient to answer the query. To provide fault tolerance through source redundancy, however, the system uses $m_{s}$ sensors to return sensor results to the cluster to cope with inconsistent readings. All sensors have the same power transmission level and have the same one-hop radio range $r$. Whether a single-hop or a multiple-hop route is required for a sensor to communicate with its cluster head depends on the cluster size whose effect on the MTTF will be analyzed. Within a cluster we assume that a cluster election protocol such as HEED [10] is in place that will more or less achieve a perfect rotation of the cluster head among all sensors in the cluster. Thus, given that the number of sensors in a cluster is $n_{s}$, the probability that a node will become a cluster head, $p$, is equal to $1 / n_{s}$. Thus total number of clusters in the network, $N_{c}$, is given by $n / n_{s}=n p$. Assume a perfect rotation of sensor nodes within a cluster to assume the role of the cluster head, so each sensor node would consume energy at about the same rate. Then, instead of considering each individual sensor energy level, we can consider the system energy whose initial energy level is given by $E_{\text {initial }}=n E_{o}$. When the energy level of the system falls below a threshold value, say $E_{\text {threshold, }}$ the WSN is considered as having depleted its energy. 


\section{Probability Model}

We define the MTTF of a sensor data system as the average number of queries that the system can answer correctly before it fails, with the failure caused by either energy depletion or sensor faults. Let $P_{q}(k)$ be the probability that a query requires $k$ clusters to respond. Let $E_{q}(k)$ be the energy consumption of the system to answer a query that requires $\mathrm{k}$ clusters, and $R_{q}(k)$ be the query reliability for a query that requires $k$ clusters to respond. Furthermore, let the average amount of energy consumed per query be $E_{q}$, given by the expected value of $E_{q}(k)$ as:

$$
E_{q}=\sum_{k=1}^{n p} E_{q}(k) P_{q}(k)
$$

Then the average number of queries that the system is able to sustain before running out its energy is given by:

$$
N_{q}=\frac{E_{\text {initial }}-E_{\text {threshold }}}{E_{q}}
$$

Let the reliability of a query be $R_{q}$, given as:

$$
R_{q}=\sum_{k=1}^{n p} R_{q}(k) P_{q}(k)
$$

Since the system is able to answer $N_{q}$ queries before energy depletion, each with the reliability of $R_{q}$, the MTTF of the system is the expected number of queries that the system can answer without experiencing a failure with the upper bound of $N_{q}$, i.e.,

$$
M T T F=\sum_{i=1}^{N_{q}-1} i R_{q}^{i}\left(1-R_{q}\right)+N_{q} R_{q}^{N_{q}}
$$

The magnitude of $E_{q}$ and $R_{q}$ largely depends on the redundancy level used by the system to answer a query and there is a tradeoff between $E_{q}$ and $R_{q}$. In the extreme case of no redundancy, $E_{q}$ would be low at the expense of low $R_{q}$. Conversely, when excess redundancy is used $R_{q}$ would be high at the expense of high $E_{q}$. We intend to show that there is an optimal level of redundancy that would maximize the MTTF.

Below we derive $R_{q}(k)$ and later we derive $E_{q}(k)$. Let $d$ be a random variable denoting the distance between a source cluster head and the processing center. Then the number of hops between the processing center and the source cluster head, denoted by $h$, is given by:

$$
h=\frac{d}{r}-1
$$

Without loss of generality, let the source cluster head be randomly located at $\left(X_{i}, Y_{i}\right)$ in the square sensor area with $-A / 2 \leq X_{i} \leq A / 2$ and $-A / 2 \leq Y_{i} \leq A / 2$ and the processing center be located in the center of the sensor area with the coordinate at $(0,0)$. Then,

$$
\begin{aligned}
E(h) & =\int_{-A / 2}^{A / 2} \int_{-A / 2}^{A / 2}\left(\frac{\left.\sqrt{\left(X_{i}^{2}+Y_{i}^{2}\right.}\right)}{r}-1\right)\left(\frac{1}{A^{2}}\right) d X_{i} d Y_{i} \\
& =\frac{0.3825 A}{r}-1
\end{aligned}
$$

For notational convenience, let

$$
N_{\text {inter }}^{h}=\lceil E(h)\rceil
$$

Here $N_{\text {inter }}^{h}$ represents the average number of hops (or sensors) to forward sensor data from a source cluster head to the processing center. Then, the failure probability of that source cluster failing to send data to the processing center, when there is a single path from the cluster head to the processing center, is given by:

$$
P_{f p}=1-(1-q)^{N_{\text {inter }}^{h}}
$$

For fault tolerance, we consider path redundancy and source redundancy. For path redundancy, let there be $m$ disjoint paths between a source cluster head and the processing center. A source cluster head can deliver the requested sensor data to the processing center if any of the $m$ redundant paths is alive. Thus, the failure probability of that source cluster failing to deliver data to the processing center can be calculated as the probability that all $m$ paths have failed. As a result, the failure probability of a source cluster failing to deliver data due to path failure, when there are $m$ disjoint paths between it and the processing center, is given by:

$$
P_{f p}^{m}=\left(P_{f p}\right)^{m}
$$

For source redundancy, instead of using one sensor, we assign $m_{s}$ sensors in each cluster to return sensor readings to their cluster head to cope with incorrect readings and sensor faults. Since a sensor becomes a cluster head with probability $p$ and all the sensors are distributed in the area in accordance with a spatial Poisson process with intensity $\lambda$, the cluster heads and non-cluster head sensors will also be distributed in accordance with a spatial Poisson process with rates $\mathrm{p} \lambda$ and (1-p) $\lambda$, respectively. Non-cluster-head sensors thus would join the cluster of the closest cluster head to form a Voronoi cell [11] corresponding to a cluster in the WSN. It can be shown that [12] the average number of non-cluster-head sensors in each Voronoi cell is (1-p)/p and the average distance from a non-cluster-head sensor to the cluster head is given by $\frac{1}{2(p \lambda)^{1 / 2}}$.

If this distance is more than per-hop distance $r$, a sensor will take a multi-hop route to transmit sensor data to the cluster head. The average number of intermediate sensors (including the sensor itself) is the quantity above divided by per-hop distance $r$. Let $N_{\text {intra }}^{h}$ denote the average number of hops to forward sensor data from a 
sensor responsible for a reading to its cluster head. Then $N^{h}{ }_{\text {intra }}$ is given by:

$$
N_{\text {int } r a}^{h}=\left\lceil\frac{1}{2 r(p \lambda)^{1 / 2}}\right\rceil
$$

A sensor will fail to return its reading to the cluster head when any hop fails, so the probability of a sensor failing to return its reading to the cluster head is given by:

$$
1-(1-q)^{N_{\mathrm{int} r a}^{h}}
$$

Consequently the failure probability that all $m_{s}$ sensors within a cluster fail to return sensor reading to the cluster head is given by:

$$
p_{f s}^{m_{s}}=\left[1-(1-q)^{N_{\mathrm{int} r a}^{h}}\right]^{m_{s}}
$$

Combining results from above, the failure probability of a cluster not being able to return a correct response, because of either path or source failure, or both, is given by:

$$
P_{f_{p s}}=1-\left(1-P_{f p}^{m}\right)\left(1-P_{f s}^{m_{s}}\right)
$$

Suppose that the application demands $k$ source clusters to return sensor data to answer a query. Also suppose that the query is considered failed when any of the $k$ clusters fails to deliver data. The overall query failure probability is given by:

$$
P_{f}=1-\left(1-P_{f_{p s}}\right)^{k}
$$

Therefore, the reliability of a query that requires $k$ clusters to respond is given by:

$$
R_{q}(k)=1-P_{f}
$$

For the energy model, we adopt the radio model in [10]. The energy used for communication is denoted as $E_{\text {elec }}$ per bit. Thus the energy spent by a sensor node to sense (or to receive) and transmit a data packet of length $n_{b}$ bits is given by:

$$
E_{\text {packet }}=2 n_{b} E_{\text {elec }}
$$

In response to a query, a sensor assigned would transmit a data packet to their respective source cluster head. Since the average number of hops between a sensor and its cluster head is given by $N^{h}{ }_{\text {intra }}$ as derived above, the average energy for the system to transmit sensor data from a sensor to its cluster head is given by $E_{\text {packet }} N^{h}{ }_{\text {intra }}$. In general if a query requires the use of $k$ clusters, each of which with $m_{s}$ sensors for source redundancy, to respond to the query, then the total energy required for these sensors within $k$ clusters to gather and forward data to their cluster heads is given by $E_{s}=E_{\text {packet }} N_{\text {intra }}^{h} k m_{s}$. Let $E_{c h}$ be the total energy consumed by the WSN to transmit sensor data from $k$ source cluster heads to the processing center for the case in which $m=1$. Since the average number of hops from the cluster head of a source cluster to the processing center is given by $N_{\text {inter }}^{h}$ derived eralier, following a similar derivation for $E_{s}, E_{c h}$ is given by $E_{c h}=$ $E_{\text {packet }} N_{\text {inter }}^{h} k$. Thus the amount of energy spent by the system, $E_{q}(\mathrm{k})$, to answer a query that demands $k$ clusters to respond, each with $m$ disjoint paths connecting the cluster head to the processing center for path redundancy is given by:

$$
E_{q}(k)=m E_{c h}+E_{s}
$$

Our objective is to find the best redundancy level represented by $m$ and $m_{s}$ that maximizes MTTF when given a set of system parameter values characterizing the application and network conditions.

\section{$4 \quad$ Numeric Results}

We exemplify the utility of analytical results derived in Section 3 with a WSN characterized by a set of model parameter values as adopted from [9] with $n=1000$ nodes, $r=1, \lambda=10$ nodes/square unit, $A=10$ units, $n_{b}=$ 50 bytes, $E_{\text {elec }}=50 \mathrm{~nJ} / \mathrm{bit}, E_{o}=2$ Joule, $E_{\text {threshold }}=0, n_{s}$ ranges from 10 to 100 nodes, $q$ ranges from $10^{-8}$ to $10^{-3}, \mathrm{~m}$ is in the range of 1 to 4 and $m_{s}$ is in the range of 1 to 7 . We intend to illustrate the tradeoff between fault tolerance and energy conservation on the MTTF metric. To simplify the analysis, we assume $P_{q}(k)=1$ for a fixed $k$ value. When $k$ is equal to $n p$, it means that all clusters are required to respond to each query.

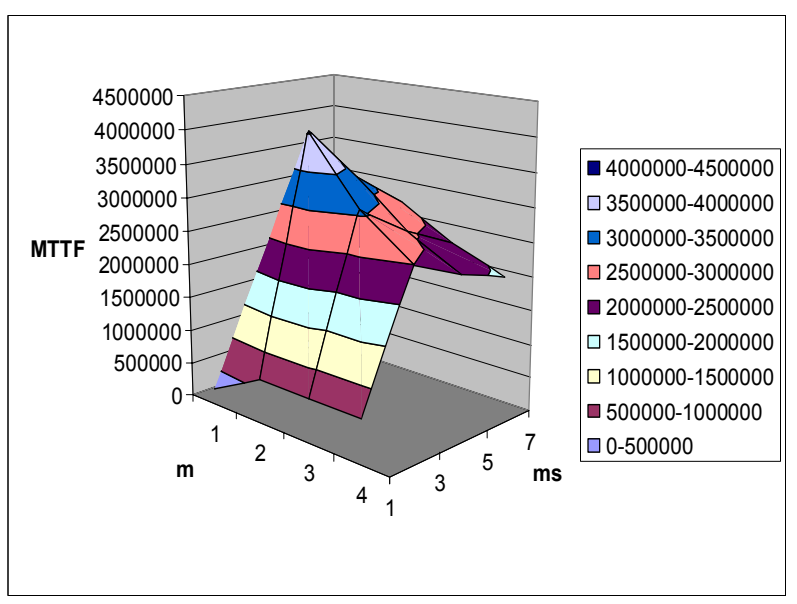

Figure 2: MTTF vs. $\left(\mathrm{m}, \mathrm{m}_{\mathrm{s}}\right)$ with $\mathrm{k}=1, \mathrm{p}=0.01$ and $\mathrm{q}=10^{-6}$.

We first demonstrate that there exists an optimal setting of $\left(\mathrm{m}, \mathrm{m}_{\mathrm{s}}\right)$ that would maximize MTTF of the WSN when given a set of parameter values characterizing the operating and workload environment. Figure 2 shows MTTF (z-coordinate) in terms of the number of queries before failure vs. $\mathrm{m}_{\mathrm{s}}$ (x-coordinate) vs. $\mathrm{m}$ (y-coordinate) in a 3-D graph for the case in which only one cluster (i.e., $k=1)$ is required to respond to a query, and the per-node failure probability $q$ is $10^{-6}$. The results show that there 
exists an optimal combination of $\left(\mathrm{m}=2, \mathrm{~m}_{\mathrm{s}}=3\right)$ where the system MTTF is maximized.

Next we test the effect of $q$. We observed that when $q$ is extremely low $\left(10^{-8}\right)$, the system would favor $m=1$ (i.e., no path redundancy) as well as $m_{s}=1$ (i.e., no source redundancy) because the chance of path or source failure is low, so the system can prolong its lifetime by not to waste energy on redundant paths and redundant sources. As the per-node failure probability increases (from $10^{-8}$ to $10^{-6}$ to $\left.10^{-3}\right)$, the system then favors a higher $\left(\mathrm{m}, \mathrm{m}_{\mathrm{s}}\right)$ combination (from 1,1 to 2,3 to 3,3 correspondingly). The reason is that as $q$ increases, the system will experience a higher probability of path failure as well as source failure, and thus the investment in energy to allow redundant paths and redundant sources pays off in prolonging the system's lifetime.

Finally we test the effect of $p$, the probability of a sensor becoming a cluster head. For the case $k=1$, we observe that as $p$ increases, or, equivalently, as the cluster size $(1 / p)$ decreases, MTTF increases. The reason is that fewer sensors would be involved in answering a query in a cluster as $p$ increases. For the case $k=n p$, that is, all clusters must respond to the query we see the opposite trend. That is, as $p$ increases, MTTF decreases. This is because as $p$ increases, they will be more clusters in the system and since all clusters must respond to the query, more energy is consumed per query since cluster heads consume more energy than regular sensor nodes.

\section{Conclusion}

In this paper we analyzed the intrinsic tradeoff between fault tolerance and energy conservation for prolonging the lifetime of WSNs designed to answer user queries. We define the system failure as the inability of the system to answer queries due to either sensor faults or energy depletion. By means of a probability model, we showed that while using path and source redundancy could increase the probability that data are delivered reliably, there is a tradeoff in reliable data delivery vs. energy consumption. We demonstrated that there exists an optimal level of redundancy that should be used by the system in order to maximize the mean time to failure, when given a set of parameter values characterizing the WSN and workload environment. Once the optimal path and source redundancy levels are determined by the system designer at static time, they can be deployed in the WSN to prolong the lifetime of the system.

\section{References}

[1] A. Badri, F. Gu and A. Ball, "Fault-Tolerant Sensor Systems: Alternative Approaches and the Development of a Complex Practical Demonstrator," Maintenance And Reliability Conference, 2001.

[2] J.Y. Chen, Y.S. Shue, H. Ogunleye and S. Bagchi, "A Comparative Study on Data Fault Tolerant
Requirements for Data Propagation in Sensor Networks," International Conference on Dependable Systems and Networks, June 2003.

[3] D. Ganesan, R. Govindan, S. Shenker and D. Estrin, "Highly-Resilient, Energy-Efficient Multipath Routing in Wireless Sensor Networks," ACM Mobile Computing and Communications Review, Vol. 5, No. 4, 2001, pp. 11-25.

[4] C. Intanagonwiwat, R. Govindan, and D. Estrin. "Directed diffusion: A scalable and Robust Communication Paradigm for Sensor Networks," ACM Mobicom, Boston, MA, 2000.

[5] G. Khanna, S. Bagchi and Y.S. Wu, "Fault Tolerant Energy Aware Data Dissemination Protocol in Sensor Networks, "International Conference on Dependable Systems and Networks, June 2004.

[6] B. Krishnamachari and S.S. Iyengar, "Efficient and Fault-Tolerant Feature Extraction in Wireless Sensor," LNCS 2634, F. Zhao and L. Guibas (Eds.), 2003, pp. 488-501.

[7] B. Krishnamachari and S.S. Iyengar, "Distributed Bayesian Algorithms for Fault-Tolerant Event Region Detection in Wireless Sensor Networks," IEEE Transactions on Computers, Vol. 53, No. 3, March 2004, pp. 241-250.

[8] F. Koushanfar, M. Potkonjak, and A. SangiovanniVincentell, "Fault Tolerance Techniques for Wireless Ad Hoc Sensor Networks," 1st IEEE International Conference on Sensors, June 2002, pp. 1491-1496.

[9] S. Bandyopadhyay, and E. Coyle, "An Energy Efficient Hierarchical Clustering Algorithm for Wireless Sensor Networks", Proc. IEEE INFOCOM, April 2003, pp. 1713-1723.

[10] O. Younis and S. Fahmy, "HEED: A Hybrid Energy Efficient, Distributed Clustering Approach for Ad Hoc Sensor Network", IEEE Transaction on Mobile Computing, Vol. 3, No. 3, October-December 2004, pp. 366-379.

[11]A. Okabe, B. Boots, K. Sugihara, and S.N. Chiu, Spatial Tessellations: Concepts and Applications of Voronoi Diagrams, $2^{\text {nd }}$ Edition, John Wiley, 2000.

[12]S.G. Foss and S.A. Zuyev, "On A Voronoi Aggregative Process Related to a Bivariate Poisson Process," Advances in Applied Probability, Vol. 28, No. 4, 1996, pp. 965-981.

[13]W. Heinzelman, C. Chandrakasan and H. Balakrishnan, "An Application-Specific Protocol Architecture for Wireless Microsensor Networks," IEEE Transactions on Wireless Communication, Vol. 1, No. 4, 2002, pp. 660-670.

[14]P. Mhatre, et al. "A Minimum Cost Heterogeneous Sensor Network with a Lifetime Constraint," IEEE Transactions on Mobile Computing, Vol. 4, No. 1, 2005, pp. 4-15. 